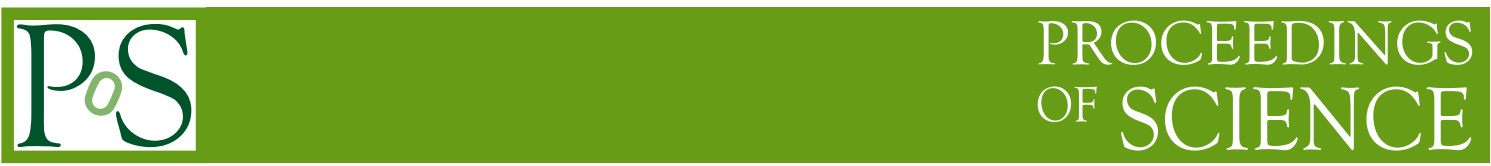

\title{
Results of NEMO-3 and Status of SuperNEMO
}

\author{
Pawel Guzowski*i \\ University of Manchester, School of Physics and Astronomy, Oxford Road, Manchester, \\ M13 9PL, United Kingdom \\ E-mail: pawel.guzowski@hep.manchester.ac.uk
}

\begin{abstract}
The NEMO-3 and SuperNEMO experiments aim to search for neutrinoless double beta decay. If observed, this would show that the neutrino is its own antiparticle, and be the the first evidence of lepton number violation. The unique setup of the experiments, using a tracker-calorimeter technique, is able to measure the full kinematic signature of the decays. This will help in determining the underlying physical process behind neutrinoless double beta decay, once it is discovered. The NEMO-3 experiment took data from 2003 to 2011 , with $7 \mathrm{~kg}$ of ${ }^{100} \mathrm{Mo}$ and $1 \mathrm{~kg}$ of ${ }^{82} \mathrm{Se}$ as the main double beta decay sources. It measured the world-leading values of the two-neutrino double beta decay half-lives for 7 isotopes. There were no neutrinoless double beta decays observed, with half-life limits set at $1.0 \times 10^{24}$ years for ${ }^{100} \mathrm{Mo}$ and $3.2 \times 10^{23}$ years for ${ }^{82} \mathrm{Se}$. The SuperNEMO experiment will improve on the NEMO-3 technique, using $100 \mathrm{~kg}$ of ${ }^{82} \mathrm{Se}$ source, and running under significantly lower background conditions with improved energy resolution. A half-life sensitivity of about $10^{26}$ years (effective neutrino mass sensitivity of about $50 \mathrm{meV}$ ) can be achieved. A demonstrator module of the experiment is currently under construction, with data taking due to begin in 2015.
\end{abstract}

The European Physical Society Conference on High Energy Physics

18-24 July, 2013

Stockholm, Sweden

\footnotetext{
* Speaker.

${ }^{\dagger}$ On behalf of the NEMO-3 and SuperNEMO collaborations
} 


\begin{tabular}{rcccc}
\hline Isotope & Abundance $(\%)$ & $Q_{\beta \beta}(\mathrm{MeV})$ & $G_{0 v}\left(10^{-14} \mathrm{y}^{-1}\right)$ & $M_{0 v}$ \\
\hline${ }^{48} \mathrm{Ca}$ & 0.19 & 4.724 & 6.35 & $0.85-2.37$ \\
${ }^{76} \mathrm{Ge}$ & 7.8 & 2.039 & 0.62 & $2.85-7.24$ \\
${ }^{82} \mathrm{Se}$ & 9.2 & 2.996 & 2.70 & $2.64-6.46$ \\
${ }^{96} \mathrm{Zr}$ & 2.8 & 3.348 & 5.63 & $1.56-5.65$ \\
${ }^{100} \mathrm{Mo}$ & 9.6 & 3.035 & 4.36 & $3.10-7.77$ \\
${ }^{116} \mathrm{Cd}$ & 7.6 & 2.809 & 4.62 & $2.51-4.72$ \\
${ }^{130} \mathrm{Te}$ & 34.5 & 2.530 & 4.09 & $2.65-5.50$ \\
${ }^{136} \mathrm{Xe}$ & 8.9 & 2.462 & 4.31 & $1.71-4.20$ \\
${ }^{150} \mathrm{Nd}$ & 5.6 & 3.367 & 19.2 & $1.71-3.70$ \\
\hline
\end{tabular}

Table 1: Isotope abundance, $Q$-value, phase space factor and nuclear matrix elements for isotopes used in the NEMO experiments, and also the competing germanium- and xenon-based experiments. From [4].

\section{Introduction}

Neutrinoless double beta decay $(0 v \beta \beta)$ is a hypothetical lepton number violating (LNV) process. There are several mechanisms which could contribute to mediating a $0 v \beta \beta$ decay. The most commonly discussed process is the Majorana mass mechanism [1]. If the neutrino is its own antiparticle (a Majorana fermion), the two daughter neutrinos from a standard double beta decay $(2 v \beta \beta)$ could virtually annihilate with each other, making the overall decay neutrinoless. However there are other LNV processes which could contribute, for example Majoron emission [2], or a right-handed weak current [3]; more examples are given in [4]. These processes can be distinguished from the Majorana mass mediated process through kinematics. For example, the right-handed current has the opposite angular distribution to the Majorana mass mediated process, as it is a $\mathrm{V}+\mathrm{A}$ interaction (where the standard weak interaction is V-A). The Majoron emission process would have a different energy spectrum, as the Majoron would carry away energy.

The NEMO-3 experiment and its successor SuperNEMO are designed to detect this decay process, and uniquely amongst the $0 v \beta \beta$ experiments, would be able to determine (or exclude) the mechanism by measuring the event kinematics. Further details on NEMO-3 are in Section 2, and on SuperNEMO in Section 3.

The half-life $T_{1 / 2}^{0 v}$ of an isotope, assuming that $0 v \beta \beta$ is mediated by the Majorana mass mechanism, is given by

$$
\frac{1}{T_{1 / 2}^{0 v}}=G_{0 v}\left(Q_{\beta \beta}^{5}, Z\right)\left|M_{0 v}\right|^{2} \frac{\left\langle m_{v}\right\rangle^{2}}{m_{e}^{2}},
$$

where $G_{0 v}$ is the phase space factor, $Q_{\beta \beta}$ is the $Q$-value of the decay, $Z$ is the atomic number, $M_{0 v}$ is the nuclear matrix element for the transition, $m_{e}$ is the electron mass, and $\left\langle m_{v}\right\rangle$ is the effective mass of the electron neutrino. Values for $G_{0 v}, M_{0 v}$, and $Q_{\beta \beta}$ for the isotopes used in the NEMO experiments are given in Table 1. When calculating the effective neutrino mass given a measured half-life, the largest uncertainty is due to the large differences in the nuclear matrix elements from various theoretical models. To achieve the best sensitivity to the $0 v \beta \beta$ half-life, isotopes with high values of $G$ and $M$ are preferred, and large $Q$-values are also advantageous, as they allow better 


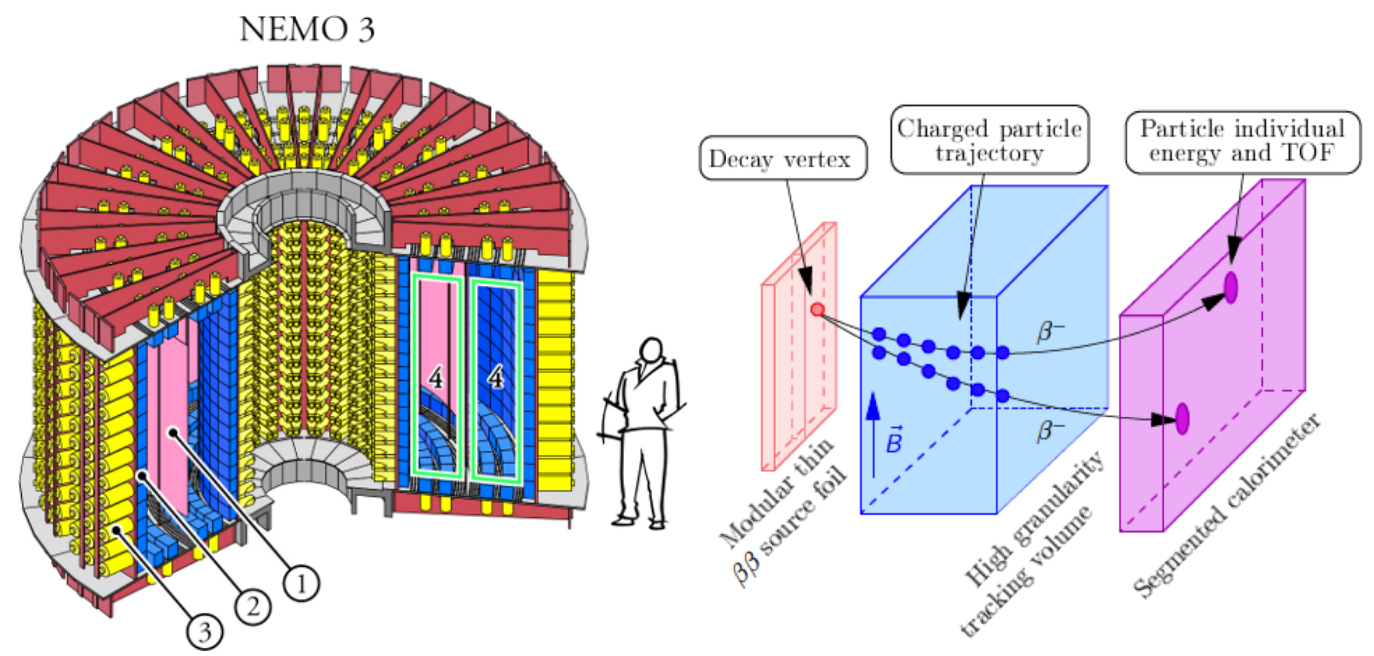

Figure 1: (L) Layout of the NEMO-3 detector: (1) source foils; (2) calorimeter blocks; (3) PMTs; (4) tracker. (R) Schematic showing the principle of operation of the detector.

background rejection. One of the unique features of the NEMO experiments is the independence of the source and the detector, allowing a wide variety of isotopes to be studied, with different values of $G$ and $M$.

\section{NEMO-3}

The NEMO-3 experiment ran from 2003 to 2011, and was located at a depth of $4.8 \mathrm{~km}$ water equivalent, in the Laboratoire Souterrain de Modane on the French-Italian border. The detector was a cylindrical structure of $3 \mathrm{~m}$ height and $4 \mathrm{~m}$ diameter (see Figure $1(\mathrm{~L})$ ). Source isotope foils were placed vertically at a radius of $1.5 \mathrm{~m}$. The experiment used $6.9 \mathrm{~kg}$ of ${ }^{100} \mathrm{Mo}, 0.9 \mathrm{~kg}$ of ${ }^{82} \mathrm{Se}$, and smaller amounts of ${ }^{130} \mathrm{Te},{ }^{116} \mathrm{Cd},{ }^{150} \mathrm{Nd},{ }^{96} \mathrm{Zr}$ and ${ }^{48} \mathrm{Ca}$. Surrounding the source foils was the tracker volume, a drift wire chamber. This was constructed from 6,180 wire cells which operated in Geiger-mode. The cells were arranged in 9 layers per side of the foil, in groups of 4, 2 and 3 layers. A $25 \mathrm{G}$ magnetic field, parallel to the cylindrical axis, permeated the tracker volume. Calorimetry was performed by 1,940 plastic scintillator blocks, coupled to 3- and 5-inch PMTs. These were arranged on the inner and outer walls of the detector, $0.5 \mathrm{~m}$ from the foil, and also on the top and bottom walls, between the tracker wire layer groups. Further details about the construction and operation of the detector are given in [5].

The principle of operation of the detector is illustrated in Figure $1(\mathrm{R})$. When an isotope double beta decays in the source foil, the decay electrons travel through the tracker, and enter the calorimeter. The calorimeter measures the energy of the electrons, along with their time of arrival. The timing between the two electrons is used to ensure they came from the source foil, and not as a result of an external background. The tracker measures the trajectory of the electrons, and using the magnetic field and the curvature of the tracks, their charge can be determined. The electron trajectories are also used to determine the location of the decay vertex, and to ensure both electrons came from the same vertex. The use of the tracker to measure the event kinematics is a unique 


\begin{tabular}{rcc}
\hline Isotope & Measured $2 v \beta \beta$ half-life $\left(10^{19} \mathrm{y}\right)$ & Publication \\
\hline${ }^{100} \mathrm{Mo}$ & $0.711 \pm 0.002$ (stat) \pm 0.054 (syst) & {$[6]$} \\
${ }^{82} \mathrm{Se}$ & $9.6 \pm 0.1$ (stat) \pm 1.0 (syst) & {$[6]$} \\
${ }^{116} \mathrm{Cd}$ & $2.88 \pm 0.04$ (stat) \pm 0.16 (syst) & \\
${ }^{130} \mathrm{Te}$ & $70 \pm 9$ (stat) \pm 11 (syst) & {$[7]$} \\
${ }^{150} \mathrm{Nd}$ & $0.911 \pm 0.025$ (stat) \pm 0.063 (syst) & {$[8]$} \\
${ }^{96} \mathrm{Zr}$ & $2.35 \pm 0.14$ (stat) \pm 0.16 (syst) & {$[9]$} \\
${ }^{48} \mathrm{Ca}$ & $4.4 \pm 0.5$ (stat) \pm 0.4 (syst) & \\
\hline
\end{tabular}

Table 2: NEMO-3 2v $\beta \beta$ half-life measurements.

feature of NEMO-3 that allows determination of the $0 v \beta \beta$ mechanism, and also offers excellent background rejection.

The main background to the $0 v \beta \beta$ search are irreducible $2 v \beta \beta$ decays. These have the same topological signature as $0 v \beta \beta$, with the only difference being that the two neutrinos carry away energy, and so the sum of the electron energies is significantly lower than the $Q$-value of the decay, with only the endpoint appearing in the signal energy range. The main reducible backgrounds are contamination of the foil and detector materials, and radon in the detector. The foil can be contaminated with ${ }^{208} \mathrm{Tl}$ and ${ }^{214} \mathrm{Bi}$, which can mimic the $0 v \beta \beta$ signal when they beta decay, and a second electron is produced for example by Compton scattering of a deexcitation photon in the foil. Impurities in the detector materials (e.g. the tracker wires or PMT glass) can produce gamma radiation, which also can induce two electrons to be produced in the source foil and mimic the signal. Radon present in the volume of the detector can leave deposits of daughter isotopes on the internal surfaces, and these daughters can similarly mimic the signal when they decay. Other radioactive backgrounds, such as alpha or gamma decays, can be easily distinguished by their topological signature. The levels of these backgrounds are all measured experimentally using the detector itself.

NEMO-3 has made the world's best measurements of the $2 v \beta \beta$ half-lives for multiple isotopes, and the results are given in Table 2.

The measurement of the $0 v \beta \beta$ half-life limit is made by examining the end-point of the twoelectron energy spectrum $\left(E_{\mathrm{TOT}}\right)$. Figure 2 shows this spectrum for ${ }^{100} \mathrm{Mo}(31.5 \mathrm{~kg} \cdot \mathrm{y}$ exposure $)$ and ${ }^{82} \mathrm{Se}\left(4.5 \mathrm{~kg} \cdot \mathrm{y}\right.$ exposure); no significant excess of events is seen in the data. For ${ }^{100} \mathrm{Mo}$ the signal window is $2.8<E_{\mathrm{TOT}}<3.2 \mathrm{MeV}$; the signal efficiency in this window is $13 \%$, and a halflife limit is set at $T_{1 / 2}>1.0 \times 10^{24}$ y with $90 \%$ confidence. For ${ }^{82} \mathrm{Se}$, the signal window is $2.6<$ $E_{\mathrm{TOT}}<3.2 \mathrm{MeV}$. The signal efficiency in this window is $14 \%$, and the $90 \%$ confidence limit on the half-life is found to be $T_{1 / 2}>3.2 \times 10^{23} \mathrm{y}$. Depending on the choice of nuclear matrix element $([10,11,12,13,14,15])$ and assuming the Majorana mass mechanism for $0 v \beta \beta$, the limits on the effective neutrino mass are found to be $\left\langle m_{v}\right\rangle<0.31-0.96 \mathrm{eV}$ for ${ }^{100} \mathrm{Mo}$, and $\left\langle m_{v}\right\rangle<0.94-2.6 \mathrm{eV}$ for ${ }^{82} \mathrm{Se}$. 

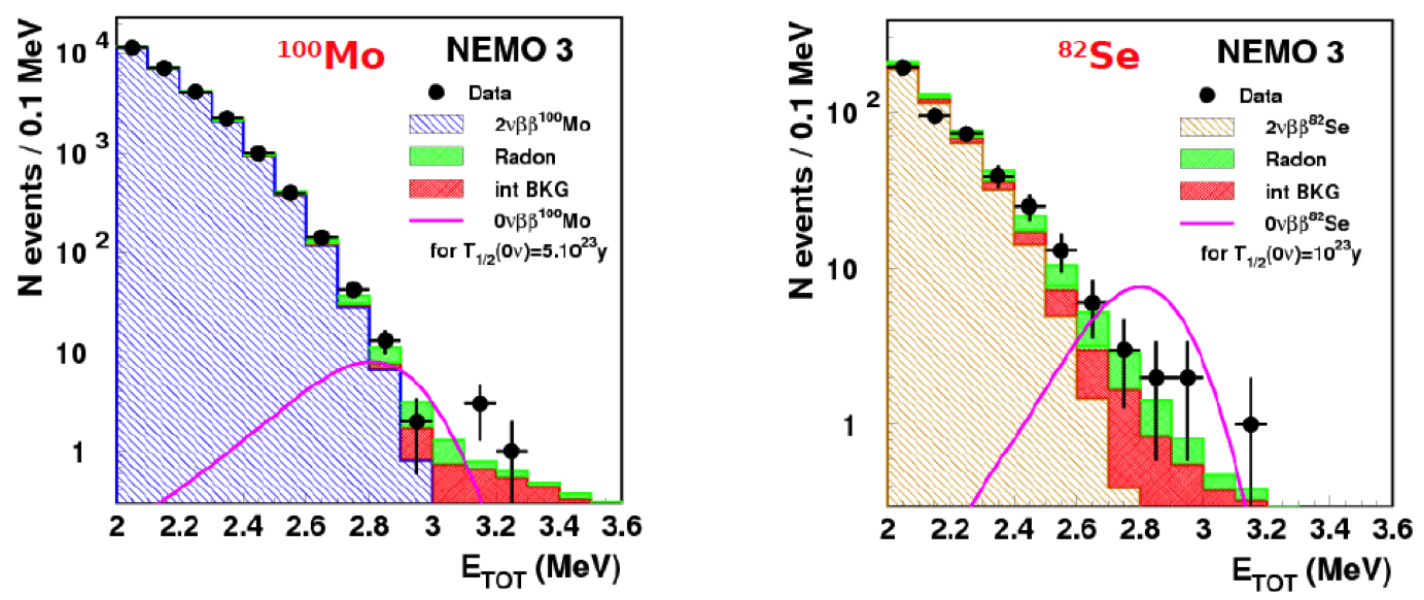

Figure 2: Spectrum of the sum of the two electron energies, for ${ }^{100} \mathrm{Mo}(\mathrm{L})$ and ${ }^{82} \mathrm{Se}(\mathrm{R})$, with the observed data as the points, and the Monte Carlo of the expected backgrounds (scaled to the measured background levels) as the filled histograms. The overlaid pink line is the spectrum for $0 v \beta \beta$ events for $T_{1 / 2}=5 \times 10^{23} \mathrm{y}$ and $T_{1 / 2}=1.0 \times 10^{23} \mathrm{y}$ respectively.

\section{SuperNEMO}

The SuperNEMO experiment is the next generation of the NEMO experimental design. Similarly to NEMO-3, the experiment will operate along the source-tracker-calorimeter principle. The whole experiment will consist of 20 modules, with each module containing around 5-10 kg of source foil in a $4 \times 3.7 \mathrm{~m}^{2}$ sheet. The modules will be planar (as opposed to the cylindrical layout of NEMO-3). A $0.5 \mathrm{~m}$ deep tracker volume will be on both sides of the foil, and one module will have around 2000 tracker cells. The calorimeter will be formed of around 500 scintillator blocks coupled to 8-inch PMTs.

To remain competitive with currently running or planned $0 v \beta \beta$ experiments, SuperNEMO must have greater than an order of magnitude lower backgrounds than NEMO-3. The source contamination of ${ }^{208} \mathrm{Tl}$ has to be less than $2 \mu \mathrm{Bq} / \mathrm{kg}$, and of ${ }^{214} \mathrm{Bi}$ less than $10 \mu \mathrm{Bq} / \mathrm{kg}$ (compared with NEMO-3 which has $\sim 100$ and $<300 \mu \mathrm{Bq} / \mathrm{kg}$ respectively). The radon level in the detector must be less than $0.15 \mathrm{mBq} / \mathrm{m}^{3}$, where in NEMO-3 it was $5 \mathrm{mBq} / \mathrm{m}^{3}$. The resolution of the calorimeter must be improved as well, doubling from a FWHM of $8 \%$ at $3 \mathrm{MeV}$ in NEMO-3, to $4 \%$ for SuperNEMO. The $0 v \beta \beta$ half-life sensitivity under these conditions will be $\sim 10^{26} \mathrm{y}$, corresponding to an effective neutrino mass of as low as $40 \mathrm{meV}$, depending on the nuclear matrix elements used.

To show that these goals can be achieved, a Demonstrator module of the detector is currently under construction. This will use $7 \mathrm{~kg}$ of ${ }^{82} \mathrm{Se}$ as the source, and $5.5 \mathrm{~kg}$ has already been produced. The foil is undergoing testing in a specially-constructed 'BiPo' detector [16], aiming to measure the ${ }^{208} \mathrm{Tl}$ and ${ }^{214} \mathrm{Bi}$ contamination levels by detecting $\beta-\alpha$ delayed coincidences. With 12 months of measurement, the BiPo detector will be sensitive to the required level of contamination. The design of the calorimeter components has been finalised, and they have been tested and shown to be within the resolution requirements. The tracker is also well into production. Each component must be radio pure, and produced under very clean conditions. One quarter of the tracker frame 
has been constructed and is currently undergoing radon emanation testing, and 36 wire cells have been produced and tested.

The Demonstrator is due to begin taking data in early 2015, operating until 2017. If the lowbackground conditions can be achieved, it should have a half-life sensitivity of $\sim 6.6 \times 10^{24} \mathrm{y}$. The construction of the remaining 19 modules will also go ahead, beginning in 2016, and the experiment is expected to run until 2021.

\section{References}

[1] S. T. Petcov, Phys. Scripta T121, 94 (2005)

[2] Y. Chikashige, R.N. Mohapatra, R.D. Peccei, Phys. Lett. B 98, 265 (1981)

[3] R. N. Mohapatra, G. Senjanovic, Phys. Rev. D 23165 (1981)

[4] W. Rodejohann, Int. J. Mod. Phys. E 20, 1833 (2011)

[5] R. Arnold et al., Nucl. Instr. Meth. A 536, 79 (2005)

[6] R. Arnold et al., Phys. Rev. Lett. 95, 182302 (2005)

[7] R. Arnold et al., Phys. Rev. Lett. 107, 062504 (2011)

[8] J. Argyriades et al., Phys. Rev. C 80, 032501 (2009)

[9] J. Argyriades et al., Nucl. Phys. A 847, 168 (2010)

[10] M. Kortelainen, J. Suhonen, Phys. Rev. C 75, 051303 (2007)

[11] M. Kortelainen, J. Suhonen, Phys. Rev. C 76, 024315 (2007)

[12] F. Simkovic, A. Faessler, V. Rodin, P. Vogel, J. Engel, Phys. Rev. C 77, 045503 (2008)

[13] J. Barea, F. Iachello, Phys. Rev. C 79, 044301 (2009)

[14] P. K. Rath, R. Chandra, K. Chaturvedi, P. K. Raina, J. G. Hirsch, Phys. Rev. C 82, 064310 (2010)

[15] E. Caurier, J. Menendez, F. Nowacki, A. Poves, Phys. Rev. Lett. 100, 052503 (2008)

[16] J. Argyriades et al., Nucl. Instr. Meth. A 622, 120 (2010) 